\title{
Avaliação do Ciclo de Vida (ACV) como recurso estratégico: uma revisão da literatura
}

\author{
Life Cycle Assessment (LCA) as \\ a Strategic factor: a literature review
}

\section{Evaluación del Ciclo de vida (ACV) como recurso estratégico: una revisión de la literatura}

\author{
JeAN Guilherme Azarias \\ Mestrando em Engenharia de Produção \\ Aparecido dos Reis Coutinho \\ Doutorado em Física \\ Professor Titular \\ Universidade Metodista de Piracicaba - UNIMEP
}

\begin{abstract}
Resumo A Avaliação do Ciclo de Vida (ACV) é uma ferramenta utilizada para analisar sistemas, seja um produto, serviço ou processo, desde o berço ao túmulo, quantificando os possíveis impactos ambientais associados a esse sistema. O presente trabalho tem por objetivo examinar a literatura que explora a utilização de ACV como um fator estratégico. Para o mapeamento da literatura existente sobre o tema, é utilizada a análise bibliométrica, que possibilita entender quantitativamente uma amostra sob diversos aspectos, como ano de publicação, origem, autoria e categorias de assunto. Uma amostra de publicações foi selecionada de acordo com critérios de inclusão e exclusão, sendo considerados apenas artigos em inglês encontrados na base de dados Scopus. O software VOSviewer foi utilizado para visualização de mapas de rede de cocitação e de conteúdo. Os padrões encontrados na amostra comprovam a aceitação da ACV como fator estratégico, dada a maior quantidade de publicações nos últimos anos. O trabalho apresenta uma análise quantitativa da literatura publicada, de forma a proporcionar um referencial teóricos para desenvolvimento de novas pesquisas nessa área.

Palavras-chave: Avaliação do Ciclo de Vida. Estratégia; revisão da literatura. Análise BIBLIOMÉTRICA.
\end{abstract}

Aвstract Life Cycle Assessment (LCA) is a tool used to analyze systems, whether a product, service, or process, from cradle to grave, quantifying the possible environmental impacts associated with this system. This study aims to examine the published literature that explores Life 
Cycle Assessment (LCA) as a strategic factor. In order to mapping the existing literature on the subject, bibliometric analysis is used, which provides a quantitative understanding of a sample under several aspects, such as year of publication, origin, authorship, and subject categories. A sample of publications was determined according to inclusion and exclusion criteria, being considered only articles in English found in the Scopus database. The VOSviewer software was used to view co-citation and content network maps. The patterns found in the article sample confirm the acceptance of LCA as a strategic factor, given the greater number of publications in recent years. This study shows a quantitative analysis of the published literature in order to provide a theoretical reference for developing further researches in this area.

Keywords: Life Cycle Assessment. Strategy. Literature review. Bibliometric analysis.

Resumen La Evaluación del Ciclo de Vida (ACV) es una herramienta utilizada para analizar sistemas, sea un producto, servicio o proceso, desde la cuna a la tumba, cuantificando los posibles impactos ambientales asociados a ese sistema. El presente trabajo tiene por objetivo examinar la literatura que explora la utilización de ACV como un factor estratégico. Para el mapeo de la literatura existente sobre el tema, se utiliza el análisis bibliométrico, que permite entender cuantitativamente una muestra bajo diversos aspectos, como año de publicación, origen, autoría y categorías de asunto. Una muestra de publicaciones fue seleccionada de acuerdo con criterios de inclusión y exclusión, siendo considerados sólo artículos en inglés encontrados en la base de datos Scopus. El software VOSviewer se utilizó para la visualización de mapas de red de cocitación y de contenido. Los patrones encontrados en la muestra comprueban la aceptación de la ACV como factor estratégico, dada la mayor cantidad de publicaciones en los últimos años. El trabajo presenta un análisis cuantitativo de la literatura publicada, de forma a proporcionar un referencial teórico para el desarrollo de nuevas investigaciones en esa área. Palabras clave: Evaluación del Ciclo de vida. estrategia; Revision de literatura. AnáLISIS BIBLIOMÉtRICO.

\section{INTRODUÇÃO}

Existe um amplo conjunto de definições para o termo "estratégia" no contexto empresarial, podendo a palavra ser definida essencialmente pelas relações dinâmicas da empresa com o ambiente, no qual deve-se tomar as ações necessárias para atingir os objetivos e/ou aumentar o desempenho por meio do uso racional dos recursos (RONDA-PUPO; GUERRAS-MARTIN, 2012). Em um contexto mais específico, consiste do planejamento e execução de mudanças competitivas executadas pelos gerentes de forma a reforçar a posição da organização no mercado, promover a satisfação dos clientes e atingir os objetivos de desempenho (THOMPSON JR; STRICKLAND III, 2000).

As ações estratégicas de uma empresa devem gerar uma vantagem competitiva, observada por uma posição mais lucrativa perante os concorrentes (PORTER, 1992) e obtida pela implementação de uma estratégia valiosa que não esteja sendo utilizada por algum concorrente 
ou potencial concorrente (BARNEY, 1991). Exemplos de vantagens competitivas de uma empresa são: proporcionar serviço superior ao cliente, ter o melhor produto disponível no mercado, obter custos menores no mercado, possuir localização geográfica privilegiada, melhor reputação do que concorrentes, entre outros (THOMPSON JR; STRICKLAND III, 2000).

No mesmo sentido, as tomadas de decisões estratégicas visam os mesmos resultados do processo de gestão estratégica, entretanto, ocorre a análise de cenários para que o mais adequado seja implementado. No que diz respeito às tomadas de decisões, existem limitações cognitivas inerentes ao tomador de decisões, sendo de grande relevância a utilização de ferramentas que possibilitem análises comparativas de cenários (EISENHARDT; ZBARACKI, 1992).

Por sua vez, o desenvolvimento sustentável (DS) tem ganhado notoriedade em instituições públicas e privadas, por representar oportunidade de consolidar ou melhorar sua posição na sociedade e no mercado, possibilitando, ainda, aumentar a geração de receita com um processo mais eficiente e com perdas reduzidas (BEVILACQUA et al., 2011; DADHICH et al., 2015).

O DS é uma prática que, em circunstâncias específicas, pode proporcionar posições únicas no mercado quando bem alinhadas, gerando vantagem competitiva em diversos departamentos da empresa, como desenvolvimento de produtos, ações de marketing, gestão de resíduos e compras verdes (HAUSCHILD et al., 2005; SALZMANN et al., 2005).

$\mathrm{O}$ desenvolvimento de atividades que atendem às necessidades do presente, sem comprometerem a capacidade das gerações futuras atenderem suas próprias necessidades, é a ideia mais clara de sustentabilidade (WCED, 1987), que deve ser considerada nas esferas ambientais, sociais e econômicas (ELKINGTON, 1998).

Com o crescimento do interesse pelo tema sustentabilidade, cresce também a quantidade de ferramentas para gerenciamento e monitoramento do DS. Dentre essas ferramentas, destaca-se a ACV - Avaliação do Ciclo de Vida, como uma forma para analisar sistemas, seja um produto, serviço ou processo, desde o berço ao túmulo, quantificando os possíveis impactos ambientais associados a esse sistema (ROBÈRT et al., 2002).

O conceito de sustentabilidade passa por um processo de amadurecimento desde a sua concepção, dando origem a formas de avaliações amplas e sistemáticas, como a ACV. Apesar de já existirem estudos sobre ACV desde a década de 1960 em alguns países, houve um crescimento na utilização e maior credibilidade advindos da norma ISO 14040 no início do século XXI, havendo uma diversidade ainda maior de estudos na segunda década (HAUSCHILD et al., 2005; GUINÉE et al., 2011).

O processo de ACV deve seguir uma sistemática para que os resultados possibilitem planejamento ou tomada de decisão assertivos, sendo implementado seguindo as etapas de 1) definição do objetivo e escopo da avaliação 2) análise de inventário das operações 3) avaliação do impacto das operações e 4) interpretação dos resultados das etapas anteriores (ABNT, 2009).

Para Eisenhardt e Zbaracki (1992), a tomada de decisão é a atividade central do processo de implementação de uma estratégia, portanto, o levantamento de dados obtidos pela ACV pode constituir o primeiro passo para as empresas, ou cadeias de empresas, desenvolverem estratégias sustentáveis (DADHICH et al., 2015). 
Em um contexto científico, em que o volume de publicações cresce continuadamente e os pesquisadores encontram cada vez mais dificuldades em rastrear literatura relevante em seu campo de estudo, a construção de um referencial teórico por meio de bibliometria proporciona uma análise quantitativa das publicações, assim como, uma visão da literatura em estudo, encontrando relações entre os elementos estruturais da amostra, como referências, autores, palavras-chave e região (ZUPIC; ČATER, 2015).

Assim, o presente artigo visa identificar as características globais da literatura publicada que associa ACV como fator estratégico, de forma a apresentar a estrutura do campo de pesquisa e tendências que proporcionem uma base para futuros trabalhos.

\section{Procedimento metodológico}

A abordagem metodológica empregada no presente trabalho consiste de uma revisão da literatura, realizada por meio de técnicas de análise bibliométrica em uma amostra de publicações. A Figura 1 apresenta o fluxograma da pesquisa realizada.

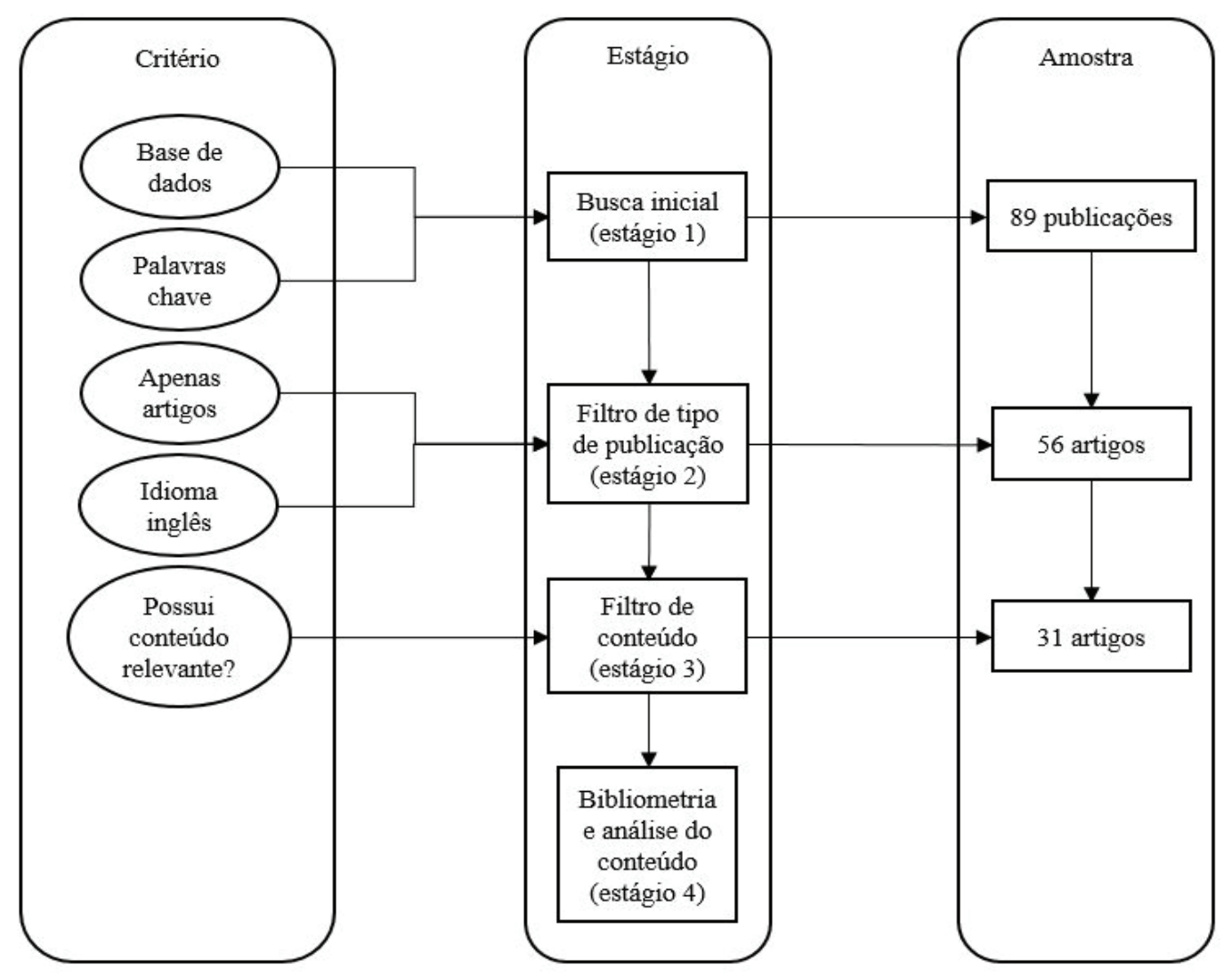

Figura 1 - Fluxograma de pesquisa.

Fonte: Adaptado de Mok (2015) 
Foram utilizadas palavras-chave para buscas na base de dados $S c o p u s$, pois apresenta conteúdo relevante para algumas áreas, como as engenharias (ZUPIC e ČATER, 2015). Foram pesquisados apenas artigos em inglês que continham a palavra "strateg*" no título e resumo, associando aos artigos com "life cycle assessment" como palavra-chave, sem restrição quanto ao período.

Foi utilizado o software VOSviewer, de acesso livre, como ferramenta de apoio à análise bibliométrica, auxiliando na identificação dos dados referentes aos autores, periódicos, origem, citações, entre outros (VAN ECK e WALTMAN, 2010). No que se refere à análise de relevância das publicações, foi considerada a quantidade de citações na base de dados Scopus.

A análise de conteúdo ocorreu nas seguintes fases: pré-análise, por meio da limitação e a definição da amostra a ser utilizada; exploração do material bibliográfico; tratamento dos resultados (BARDIN, 2008), sendo conduzida mediante análise de frequência das palavras compartilhadas entre os artigos (DELECROIX e EPPSTEIN, 2004; GENG et al., 2017), bem como, por leitura extensiva dos artigos da amostra.

\section{Resultados e discussão}

A amostra inicial, obtida apenas pela busca com as palavras na base de dados, resultou em uma Quantidade de Publicações igual a 89 e, após a aplicação dos filtros de idioma e formato da publicação, foram reduzidas para 56 artigos, dos quais 31 foram selecionados por possuírem conteúdo dentro do escopo da pesquisa.

Os estudos encontrados na amostra indicam que a ACV começou a ser associada com alguma forma de estratégia a partir de 1995, porém a parte mais representativa das publicações encontra-se entre os anos de 2010 e 2017.

\subsection{Distribuição por categorias de assunto}

Os artigos encontrados foram classificados pela base de dados Scopus em 16 categorias de assuntos diferentes, conforme a Figura 2, podendo haver artigos classificados em mais de um assunto.

Como principal categoria de assunto se destaca a Ciência Ambiental, com 71\% dos artigos, mostrando o foco da ACV como ferramenta para a gestão ambiental. Em segundo lugar aparece o assunto de Engenharia (38,7\% dos artigos) que, com os assuntos de Negócio, Gestão e Contabilidade (32,3\%) e Ciências da Decisão (6,5\%), possui uma relação mais próxima com o tema de estratégias. Voltando ao tema de gestão ambiental, observa-se que 35,5\% dos artigos estão relacionados ao assunto de Energia, que fica em terceiro lugar.

Ainda, algumas outras categorias de assunto menos relacionadas à gestão ambiental e estratégica aparecem na amostra, por exemplo, Agricultura e Ciências Biológicas (6,5\%), Ciências Sociais $(6,5 \%)$ e Medicina (3,2\%). 
Categoria de assunto

Ciência Ambiental

Engenharia

Energia

Negócio, Gestão e Contabilidade

Ciência da Computação

Economia, Econometria e Finanças

Agricultura e Ciências Biológicas

Ciências da Decisão

Ciências Sociais

Engenharia Química

Química

Medicina

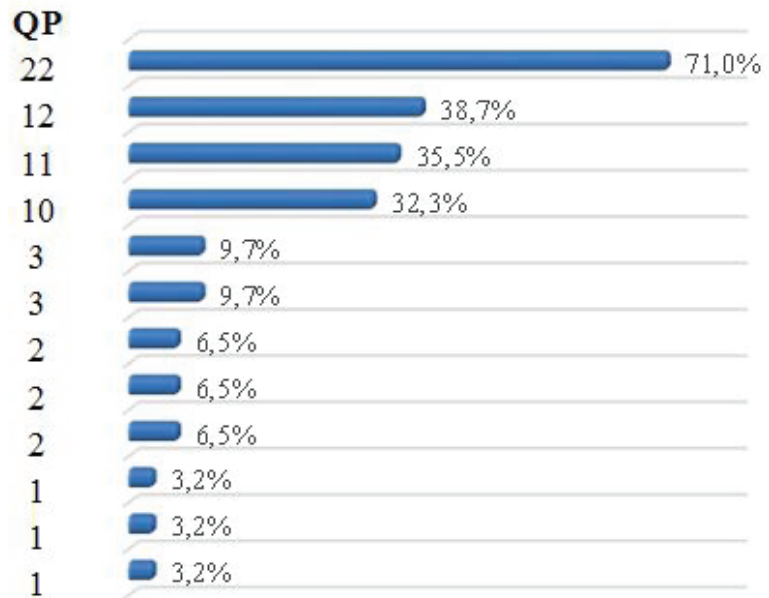

Figura 2 - Distribuição dos artigos por categorias de assunto

\subsection{Distribuição por origem}

Os artigos foram analisados quanto ao país e instituição de origem dos autores. Foram encontrados um total de 20 países, representados pela Figura 3.

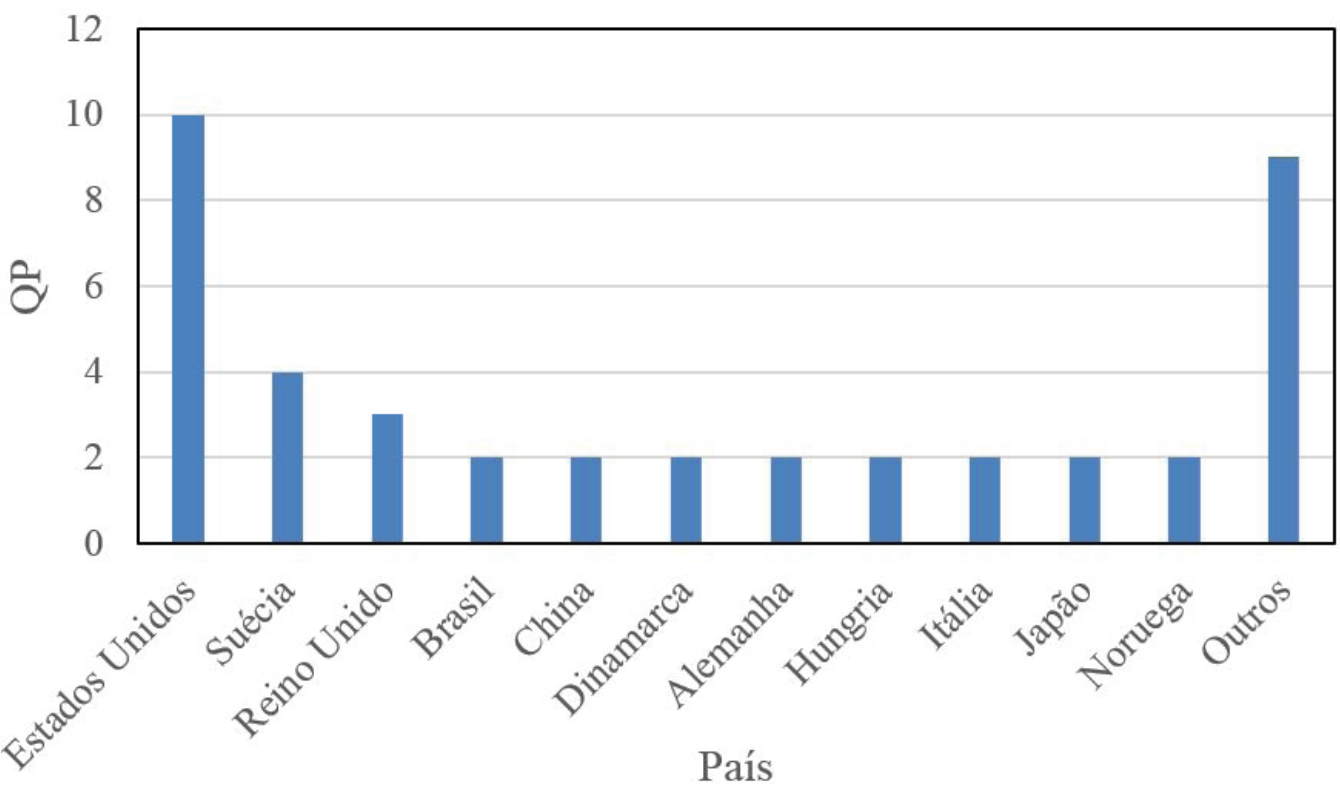

Figura 3 - Distribuição dos artigos por origem geográfica

No que diz respeito ao país de origem dos autores, um ponto importante a ressaltar é o nível de colaboração internacional entre os autores. Dentre a amostra com 31 artigos, 11 deles possuem a coautoria com diferentes nacionalidades. 
Existem nove países com a QP igual a um, representados na Figura 3 como Outros, e oito países que aparecem em duas publicações, dentre eles o Brasil. Os Estados Unidos aparecem em primeiro lugar, com dez publicações, em segundo lugar fica a Suécia, seguida do Reino Unido, em terceiro lugar.

Em relação às instituições, foram encontradas 61 instituições diferentes na amostra com alto nível de dispersão devido ao fato de existir uma grande quantidade de instituições com apenas uma publicação, destacando como exceção, a instituição norte-americana UC Berkeley, que aparece duas vezes na amostra

\subsection{Distribuição por periódico}

Foram encontrados 22 periódicos como fonte de publicação dos artigos e, a exemplo do que ocorre nas categorias de assunto, com uma ampla variedade de áreas de conhecimento, a maioria com apenas uma publicação.

Dentre os periódicos encontrados, três possuem mais de uma publicação, são eles o Journal of Cleaner Production, com oito artigos publicados na amostra, e o Resources Conservation and Recycling e Waste Management, ambos com duas publicações na amostra. Os periódicos encontrados como fonte de publicação na amostra são apresentados na Figura 4.

Journal of Cleaner Production

Resources Conservation and Recycling

Waste Management

Acta Horticulturae

Applied Energy

Building Research and Information

Business Horizons

Computers and Chemical Engineering

Ecological Indicators

Energy Policy

Environmental Impact Assessment Review

Environmental Modeling and Software

Environmental Monitoring and A ssessment

Environmental Research Letters

Environmental Science and Technology

Intelligent Decision Technologies

International Journal of Logistics Systems and Management

Journal of Industrial Ecology

Journal of Ship Production

Research in Engineering Design

Science of the Total Environmental

Water Science and Technology

TOT AL

Figura 4 - Distribuição dos artigos por periódico

\begin{tabular}{ccl} 
QP & \% & \\
8 & $25,8 \%$ & $25,8 \%$ \\
2 & $6,5 \%$ & $6,5 \%$ \\
2 & $6,5 \%$ & $6,5 \%$ \\
1 & $3,2 \%$ & $3,2 \%$ \\
1 & $3,2 \%$ & $3,2 \%$ \\
1 & $3,2 \%$ & $3,2 \%$ \\
1 & $3,2 \%$ & $3,2 \%$ \\
1 & $3,2 \%$ & $3,2 \%$ \\
1 & $3,2 \%$ & $3,2 \%$ \\
1 & $3,2 \%$ & $3,2 \%$ \\
1 & $3,2 \%$ & $3,2 \%$ \\
1 & $3,2 \%$ & $3,2 \%$ \\
1 & $3,2 \%$ & $3,2 \%$ \\
1 & $3,2 \%$ & $3,2 \%$ \\
1 & $3,2 \%$ & $3,2 \%$ \\
1 & $3,2 \%$ & $3,2 \%$ \\
1 & $3,2 \%$ & $3,2 \%$ \\
1 & $3,2 \%$ & $3,2 \%$ \\
1 & $3,2 \%$ & $3,2 \%$ \\
1 & $3,2 \%$ & $3,2 \%$ \\
1 & $3,2 \%$ & $3,2 \%$ \\
1 & $3,2 \%$ & $3,2 \%$ \\
$\mathbf{3 1}$ & $\mathbf{1 0 0 , 0} \%$ & \\
& & \\
\hline 3
\end{tabular}




\subsection{Análise dos autores mais influentes}

Por meio de uma análise de cocitação, na qual os autores mais citados pelos 31 artigos da amostra são considerados, foram encontrados 2.019 autores diferentes. A análise considera que, quando dois autores são citados pelo mesmo artigo, eles possuem uma ligação e o conjunto dessas ligações forma as correntes de autores.

A Figura 5 ilustra as redes formadas pelos autores que foram citados pelo menos cinco vezes dentro da amostra, resultando em um total de 52 autores, divididos em cinco correntes diferentes, distintas por cores. Em relação à relevância dos autores, quanto maior a circunferência, maior a quantidade de citações.

A corrente principal, representada pela cor vermelha, possui 16 autores e indica que a força de ligação entre esses autores é mais intensa do que nas demais correntes. O principal autor dessa corrente é o G. Finnveden. $\mathrm{O}$ autor mais relevante da amostra faz parte da segunda corrente de autores, representada pela cor verde, é o R. Heijungs, pesquisador na Vrije Universiteit Amsterdam - Holanda.

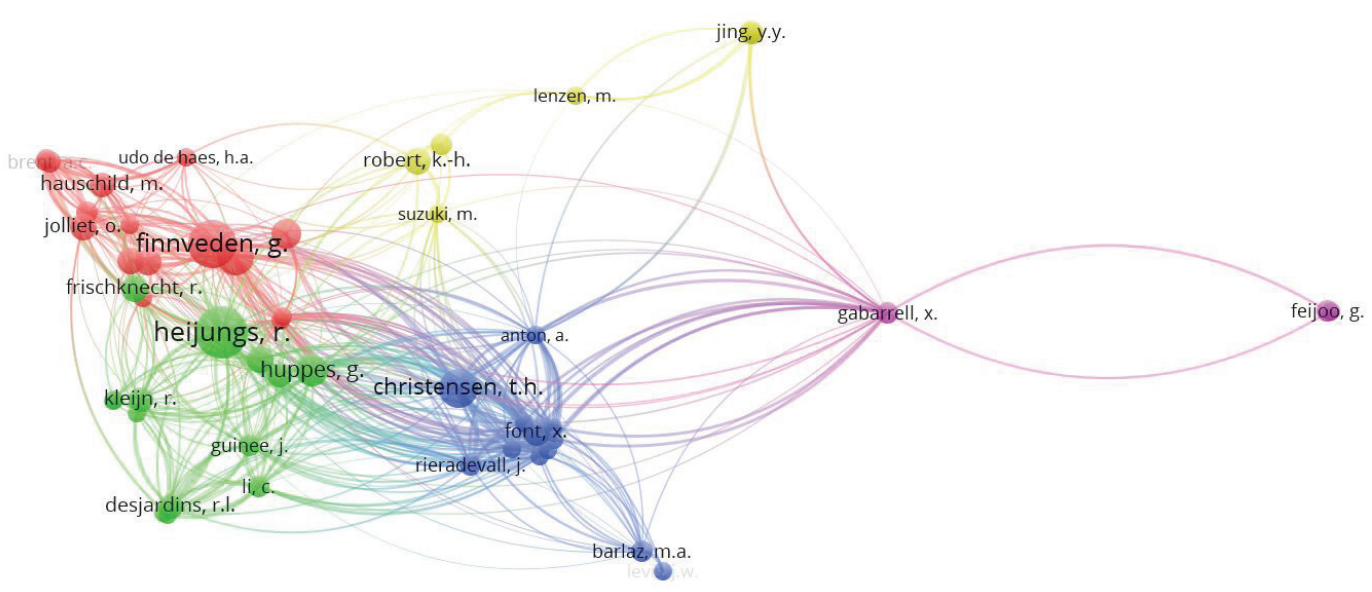

Figura 5 - Mapa de rede de cocitação

\subsection{Análise do conteúdo}

Assim como para a análise de cocitação, o recurso de mapa foi utilizado para analisar de maneira geral o conteúdo dos artigos da amostra. Foram consideradas as palavras que ocorrem no título ou resumo dos artigos com contagem binária, em que se verifica a ocorrência da palavra no artigo, não importando a frequência.

Foram encontradas 1.142 palavras diferentes e a Figura 6 apresenta apenas as 75 palavras que ocorrem em pelo menos três artigos, sendo algumas das principais: life cycle assessment, strategy, analysis, emission, sustainability, waste, production, impact, energy, global warming e treatment.

Para a corrente principal de palavras, cor vermelha, os principais temas abordados remetem à estudos referentes à implementação de ferramentas para a qualidade ambiental, bus- 
cando melhor desempenho e eficiência. A segunda corrente, cor verde, possui um foco mais voltado para a comparação entre produtos e processos, visando à redução no impacto ambiental e aquecimento global. A terceira corrente, cor azul, possui maior relação com sistemas de disposição no fim de vida, abordando temas como gerenciamento de resíduos, recuperação, descarte e tratamento.

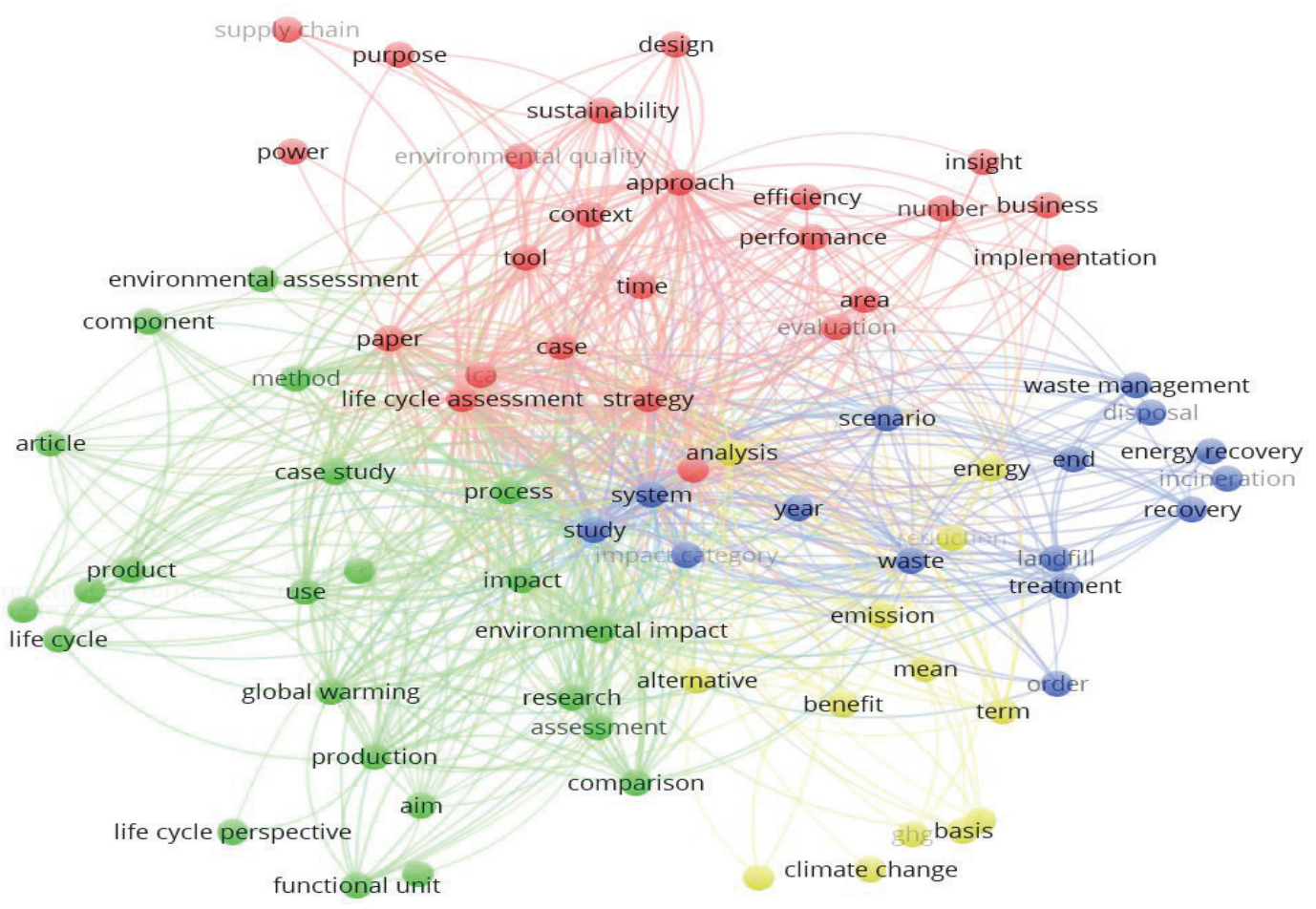

Figura 6 - Mapa de rede de coocorrência de palavras

\subsection{Tipos de estudo}

A amostra de artigos foi analisada quanto ao tipo de estudo realizado, classificados entre estudos conceituais-teóricos, analíticos (incluindo modelagem, simulação e experimentos) ou empíricos (incluindo surveys, estudos de caso e pesquisa-ação), conforme realizado por Carnevalli e Miguel (2008).

Como representado pela Tabela 1, apenas uma pequena parte dos estudos é do tipo conceitual-teórico, tendo como principal destaque, com a maior quantidade de citações da amostra, o artigo "Strategic sustainable development - selection, design and synergies of applied tools" (ROBÈRT et al., 2002), que traz uma revisão com as principais ferramentas e elementos para o DS existentes até o momento da publicação.

Como estudos analíticos, que envolvem na sua maioria proposições de modelos, foram encontrados 13 artigos, sendo sete deles acompanhados de estudos empíricos, validando o modelo proposto, por exemplo, o artigo "The environmental performance strategy map: an 
integrated LCA approach to support the strategic decision-making process" (BENEDETTO; KLEMES, 2009), que propõe uma forma gráfica de análise integrada entre alguns do principais indicadores ambientais e o custo, aplicando em uma indústria de fertilizantes e pesticidas na Dinamarca, de forma a favorecer o processo de tomada de decisões.

Dentre os artigos da amostra, a maior parte possui estudos estritamente empíricos, como no caso do "Life cycle assessment of a solar combined cooling heating and power system in different operation strategies" (JING et al., 2012), explorando a ACV como forma de análise para diferentes estratégias de operações de um sistema de aquecimento e resfriamento em um prédio comercial na China.

Tabela 1 - Distribuição da amostra por tipo de estudo

\begin{tabular}{lcc}
\hline \multicolumn{1}{c}{ Tipo de estudo } & QP & $\%$ \\
\hline Estudo empírico & 14 & 45,16 \\
Estudo analítico & 6 & 19,35 \\
Estudo conceitual-teórico & 4 & 12,90 \\
Estudo empírico + estudo analítico & 7 & 22,58 \\
\hline
\end{tabular}

\subsubsection{Indicadores avaliados}

Estudos relacionados à ACV apresentam como principal objetivo a análise dos impactos, dessa forma os artigos da amostram foram classificados entre cinco categorias de indicadores, sendo eles: pegada de carbono, de água, de energia, de emissões e do ambiente de trabalho. (BENEDETTO; KLEMES, 2009).

Conforme disponibilizado na Tabela 2, o indicador mais utilizado foi referente à emissões de gases do efeito estufa, que é objetivo de redução para muitas empresas há duas décadas (MARCOVITCH, 2014). A utilização de mais de um indicador ocorre em quatro artigos, dos quais apenas um aborda os cinco indicadores. Ainda, existem 16 artigos que abordam outros tipos de indicadores, que não contemplam um dos analisados, ou que não focam um tipo de indicador específico.

Tabela 2 - Tipos de indicadores utilizados na amostra

\begin{tabular}{lc}
\hline \multicolumn{1}{c}{ Indicador analisado } & QP \\
\hline Pegada de emissões & 13 \\
Pegada de energia & 4 \\
Pegada de carbono & 3 \\
Pegada do ambiente de trabalho & 2 \\
Pegada de água & 1 \\
Outros ou não especificado & 16 \\
\hline
\end{tabular}




\subsubsection{Aplicação dos estudos}

Como forma de analisar as aplicações dos estudos, foram classificados em quatro categorias diferentes, conforme a Tabela 4. A grande maioria dos artigos foi aplicada para análise de serviços ou produtos, com 10 e nove estudos respectivamente. O artigo "ISO 14000 as a strategic tool for shipping and shipbuilding" (FET, 1998) realiza uma análise teórica de como a utilização da norma ISO 1400, que é um pacote de normas incluindo a ACV, pode ser aplicada para otimização de políticas e tomada de decisões, tanto para produtos (construção de navios) quanto serviços (transporte) na Noruega.

Dentre os cinco artigos aplicados para a gestão de resíduos, o artigo "Allocation strategies in comparative life cycle assessment for recycling: considerations from case studies" (TONIOLO et al., 2017) é o único associado com produto, realizando uma análise comparativa entre diferentes estratégias de reciclagem para o ciclo de vida de produtos.

As publicações aplicadas para um gerenciamento estratégico da cadeia de suprimentos aparecem apenas cinco vezes, tendo ainda três artigos que não especificam o tipo de aplicação do estudo.

Tabela 3 - Tipos de aplicações utilizados na amostra

\begin{tabular}{lc}
\hline \multicolumn{1}{c}{ Aplicação do estudo } & QP \\
\hline Serviço & 10 \\
Produto & 9 \\
Cadeia de suprimento & 6 \\
Gestão de resíduos & 5 \\
Não especificado & 3 \\
\hline
\end{tabular}

\section{CONSIDERAÇÕES FINAIS}

A maioria dos artigos analisados possui os Estados Unidos como nacionalidade dos autores, assim como a principal instituição da amostra também é norte-americana. Em relação à fonte de publicação, o periódico Journal of Cleaner Production se destaca com a maior quantidade de publicações analisadas. Existe uma corrente composta por 16 autores que exercem maior influência na área em estudo, porém o autor mais citado é R. Heijungs, pertencente a outra corrente de autores.

Os estudos com abordagem empírica foram os mais encontrados na amostra analisada, sendo o principal indicador ancorado na quantidade de emissões. Apesar de a maioria dos artigos estudar o setor de serviços, a aplicação para produtos também é representativa na amostra.

Os resultados do presente trabalho poderão contribuir para que os pesquisadores possam basear suas pesquisas em revisão da literatura, tanto quantitativa quanto qualitativa, facilitando o rastreamento de literatura relevante. 


\section{Agradecimentos}

O presente trabalho foi realizado com apoio da Coordenação de Aperfeiçoamento de Pessoal de Nível Superior - Brasil (CAPES) - Código de Financiamento 001.

\section{ReferÊNCIAS}

ABNT - ASSOCIAÇÃO BRASILEIRA DE NORMAS TÉCNICAS. NBR ISO14040: Gestão ambiental - Avaliação do ciclo de vida - Princípios e estrutura. Rio de Janeiro, 2009.

BARDIN, L. Análise de conteúdo. $4^{\mathrm{a}}$ ed., Lisboa: Edições 70, 2008.

BARNEY, J. Firm resources and sustained competitive advantage. Journal of Management, vol. 17, p. 99-120, 1991.

BENEDETTO, L.; KLEMES, J. The environmental performance strategy map: an integrated LCA approach to support the strategic decision-making process. Journal of Cleaner Production, vol. 17, p. 900-906, 2009.

BEVILACQUA, M.; CIARAPICA, F. E.; GIACCHETTA, G.; MARCHET'TI, B. A carbono footprint analysis in the textile supply chain. International Journal of Sustainable Engineering, vol. 4, p. 24-36, 2011.

CARNEVALLI, J. A.; MIGUEL, P. C. Review, analysis and classification of the literature on QFD - Types of research, difficulties and benefitis. International Journal of Production Economics, vol. 114, p. 737-754, 2008.

DADHICH, P.; GENOVESE, A.; KUMAR, N.; ACQUAYE, A. Developing sustainable supply chains in the UK construction industry: a case study. International Journal of Production Economics, vol. 164, p. 271-284, 2015.

DELECROIX, B.; EPPSTEIN, R. Co-word analysis for the non-scientific information example of Reuters business briefings. Data Science Journal, vol. 3, p. 80-87, 2004.

EISENHARDT, K. M.; ZBARACKI, M. J. Strategic decision making. Strategic Management Journal, vol. 13, p. 17-37, 1992.

ELKINGTON, J. Cannibals with fork: the triple bottom life of 21 st century. Gabriola Island, BC: New Society LtPublishers, 1998.

FET, A. M. ISO 14000 as a Strategic Tool for Shipping and Shipbuilding. Journal of Ship Production, vol. 14, p. 155-163, 1998.

GENG, S.; WANG, Y.; ZUO, J.; ZHOU, Z.; DU, H.; MAO, G. Building life cycle assessment research: A review by bibliometric analysis. Renewable and Sustainable Energy Reviews, vol. 76, p. 176-174, 2017.

GUINÉE, J. B.; HEIJUNGS, R.; HUPPES, G. Life Cycle Assessment: past, presente, and future. Environmental Science \& Technology, vol. 45, p. 90-96, 2011.

HAUSCHILD, M.; JESWIET, J.; ALTING, L. From life cycle assessment to sustainable production: status and perspectives. CIRP Annals - Manufacturing Technology, vol. 54, p. $1-21,2005$. 
JING, Y. Y.; BAI, H.; WANG, J. J.; LIU, L. Life cycle assessment of a solar combined cooling heating and power system in different operation strategies. Applied Energy, vol. 92, p. 843853, 2012.

MARCOVITCH, Jacques (Org.). Estratégias Empresariais e a Redução de Emissões de Gases de Efeito Estufa (GEE). São Paulo: FEA/USP, 2014. Disponível em: http://www. usp.br/mudarfuturo/cms

MOK, K. Y.; SHEN, G. Q.; YANG, J. Stakeholder management studies in mega construction projects: A review and future directions. International Journal of Project Management, vol. 33, p. 446-547, 2015.

PORTER, M. E. Vantagem Competitiva: criando e sustentando um desempenho superior. $5^{\text {a }}$ ed., Rio de Janeiro: Editora Campus, 1992.

ROBÈRT, K. H.; SCHIMIDT-BLEEK, B.; LARDEREL, J. A.; BASILE, G.; JANSEN, J. L.; KUEHR, R.; THOMAS, P. P.; SUZUKI, M.; HAWKEN, P.; WACKERNAGEL, M. Strategic sustainable development - selection, design and synergies of applied tools. Journal of Cleaner Production, vol. 10, p. 197-214, 2002.

RONDA-PUPO, G. A.; GUERRAS-MARTIN, L. A. Dynamics of the evolution of the strategy concept 1962-2008: a co-word analysis. Strategic Management Journal, vol. 33, p. 162188, 2012.

SALZMANN, O.; IONESCU-SOMERS, A.; STEGER, U. The business case for corporate sustainability: literature review and research options. European Management Journal, vol. 23, p. 27-36, 2005.

THOMPSON JR, A. A.; STRICKLAND III, A. J. Planejamento estratégico: elaboração, implementação e execução. Pioneira, 2000.

TONIOLO, S.; MAZZI, A.; PIERETTO, C.; SCIPIONI, A. Allocation strategies in comparative life cycle assessment for recycling: considerations from case studies. Resources, Conservation and Recycling, vol. 117, p. 249-261, 2017.

VAN ECK, N. J.; WALTMAN, L. Software survey: VOSviewer, a computer program for bibliometric mapping. Scientometrics, vol. 84, p. 523-538, 2010.

WCED - World Comission on Environmental and Development. Our Common Future (Relatório de Brundtland). Oxford: Oxford University Press, 1987.

ZUPIC, I.; ČATER, T. Bibliometric methods in management and organization. Organization Research Methods, vol. 18, p. 429-472, 2015.

Submetido em: 5-2-2018

Aceito em: 25-10-2018

"O presente trabalho foi realizado com apoio da Coordenação de Aperfeiçoamento de Pessoal de Nível Superior - Brasil (CAPES) - Código de Financiamento 001”.

"This study was financed in part by the Coordenação de Aperfeiçoamento de Pessoal de Nível Superior - Brasil (CAPES) - Finance Code 001”. 